\title{
COVID-19 vaccine causing Guillain barre syndrome, a rare potential side effect
}

ahmad matarneh ${ }^{1}$, alia al-battah ${ }^{2}$, khalid farooqui ${ }^{2}$, mohamed ghamoodi ${ }^{3}$, and Mohammed alhatou $^{3}$

${ }^{1}$ Hamad medical corporation

${ }^{2}$ hamad medical corporation

${ }^{3}$ Hamad Medical Corporation

July 11, 2021

\begin{abstract}
COVID-19 Virus has affected the world in many ways, resulting in several challenges. COVID 19 vaccines were introduced recently, with a variable degree of immunogenicity and safety. We report a 61-year-old man who had GBS a few days after Receiving The vaccine, his condition improved after 5 days of IVIG
\end{abstract}

COVID-19 vaccine causing Guillain barre syndrome, a rare potential side effectAhmad S matarneh 1, Alia Hani Al-battah 2, Khalid Farooqui 1, Mohamed ghamoodi 2, mohammed alhatou 21. Internal Medicine department, Hamad medical corporation, Doha, Qatar.

2. Department of neurology, Hamad medical corportation, Doha, Qatar/

*Corresponding Author

Dr. Ahmad Samir Salam Matarneh.

Department Internal Medicine.

Hamad Medical Corporation.

Al Rayyan street.

Doha, Qatar.

Tel: +97455957396

Fax: -44397857

E-mail: AMatarneh@Hamad.qa

Keywords:

Gullain Barre syndrome, COVID 19 vaccine, Pandemic, neurology

Key Clinical message: Patients with neurological symptoms should be enquired about recent vaccination history. It is important after the COVID 19 mRNA vaccine, which is newly introduced as it might have a link to the development of a wider variety of neurological diseases

\section{Abstract:}


COVID-19 Virus has affected the world in many ways, resulting in several challenges. It has resulted in high rates of morbidity and mortality. Efforts have been made to study the nature of the disease and discover a new treatment to help decrease those rates. COVID 19 vaccines were introduced recently $\backslash$ sout, with a variable degree of immunogenicity and safety.

We report a 61-year-old man who developed GBS within 4 days of receiving the Moderna SARS-CoV-2 vaccine. Patient presented with 3-day history of, distal more than proximal, upper extremity progressive weakness four days following receiving the second dose mRNA-based vaccine. Common infectious triggers of Guillain-Barre syndrome (GBS) were ruled out. His clinical picture, CSF analysis and electrodiagnostic testing were consistent with acute demyelinating polyneuropathy. His clinical condition significantly improved after five days course of intravenous immunoglobulin (IVIG).

COVID-19 vaccines have been tested in large, randomized controlled trials. The vaccines have shown a high level of efficacy and safety across all populations. However, they can result in different side effects, as is the case with all other vaccines. Neurological side effects can occur following COVID-19 vaccines. However, their frequency is not well studied yet. One of those side effects is the development of Guillain barre syndrome. The frequency of its occurrence is still not precise, and many other studies are needed to clarify its incidence further. The temporal relationship between COVID-19 vaccination and GBS development in this case was suggestive of a vaccine-induced cause, and the clinical implications of this association warrant further research.

\section{Introduction :}

The severe acute respiratory syndrome coronavirus 2 (SARS-CoV-2) Is a newly emerging disease entity first reported in Wuhan, China, in December 2019. it led to high morbidity and mortality rates, affecting the world on the social, psychological, and financial aspects (1). its presentations are widely variable depending on the system involved. The main presenting symptoms are respiratory, ranging from asymptomatic to a more severe lower respiratory tract infection, acute respiratory distress syndrome (ARDS), and in more severe cases, death. However, it can also infect the central nervous, cardiovascular, and gastrointestinal systems (2). Numerous treatments have been utilized for treating it, and the evidence on how to treat it is growing daily. (3) since its emergence, efforts have been put to find a vaccine that would halt the progression of the pandemic. In December 2020, the first COVID-19 vaccines were successfully announced with promising results and were approved for use after an emergency authorization by FDA. (4) Side effects secondary to these vaccines were variable, and further studies are needed to establish their safety. some of the side effects described are myalgia, fever, and headache. Other side effects were reactivation of the COVID-19 and ARDS (5). neurological side effects are rare but have been described (6). We hereby report a case that we encountered after an mRNA vaccine and was found to have features of acute demyelinating polyneuropathy as found by nerve conduction studies.

\section{Case presentation:}

A 61-year-old gentleman presented to the emergency department with a 3-day history of bilateral upper limb weakness, mainly distally involving the hands, described as an inability to perform fine hand movements such as buttoning his shirt and holding the pen properly when writing. He denied having any numbness or pain. There were no other neurological deficits. There was no antecedent respiratory tract infection or diarrheal illness. No history of a similar attack before. His symptoms started four days after receiving the second dose of the COVID-19 vaccine (Moderna)

On examination, the patient had normal vital signs. Neurological examination was remarkable for

Decreased Power was $5 / 5$ in proximal muscles and $4 / 5$ in distal muscles. Both upper limbs showed no fasciculation, no abnormal movements, normal tone. He had normal deep tendon reflexes all over. The sensory and cerebellar examinations were normal. Neurological examination of the lower limbs was normal. Cardiac, respiratory, and abdominal examinations were normal. Complete blood count, urea, 
creatinine, electrolytes, C-reactive protein, procalcitonin, vitamin B12, folic acid, and thyroid function tests were normal (Table 1$)$.

Lumbar puncture was performed, CSF analysis revealed pink fluid, RBC 3000/ul, WBC 4/ul [corrected is 6 when using $1 \mathrm{WBC}$ : $500 \mathrm{RBC}$ ratio], Glucose $4.27 \mathrm{mmol} / 1$, protein $0.5 \mathrm{gm} / 1$ [normal range $0.15-0.45 \mathrm{gm} / \mathrm{l}]$ indicating albumin-cytologic dissociation. (Table 2)

Nerve conduction studies showed electrophysiological evidence of pure motor neuropathy with primary demyelinating features suggestive of demyelinating polyneuropathy. (Figure (1). The patient received IV immunoglobulin therapy total of $2 \mathrm{gm} / \mathrm{kg}$; IVIG $0.4 \mathrm{gm} / \mathrm{kg}$ per day for 5 days. His power improved throughout his hospital stay and he was discharged home.

Discussion : COVID 19 has affected the world in many ways. It created a high burden on countries and populations and currently remains a challenge as it is associated with high morbidity and mortality rates. The impact of the pandemic resulted in a considerable change in day-to-day life (7). since Its emergence, researchers have been working robustly to find a cure. COVID 19 vaccines were introduced recently after extensive work to find a method to halt the progression of this pandemic. New technology was introduced, which is the development of mRNA-based vaccines; BNT162B2 and mRNA-1273 being the primary examples. mRNA vaccines work by inducing a T-cell mediated immune response to a protein that is translated from the mRNA. They have been shown to exhibit a significant level of immunity. (8) However, data regarding the safety of these vaccines is still lacking, and further studies are needed to uncover all the potential side effects (9). It is not uncommon to have neurological side effects following vaccination, as described in the literature. (10) COVID 19 vaccines have been linked to several CNS side effects as observed in trials, as well as they are being reported in case reports. The incidence Is yet to be confirmed. As more and more people are being vaccinated, more side effects will be noticed (11). side effects that were reported included weakness, numbness, ataxia, and more severe presentations such as encephalitis, myelitis, and demyelination with Guillain barre syndrome. (12) GBS development secondary to vaccines has been described before in literature (13). however, its development after the COVID 19 vaccine is not well reported, and further studies are needed to expand on it (14).

Guillain-Barré syndrome (GBS) is an acute immune mediated disease of peripheral nerves and nerve roots that is usually preceded by respiratory or gastrointestinal infection. It presents with progressive, ascending, symmetrical limb weakness, and paresthesia with diminished or absent deep tendon reflexes, with or without respiratory and cranial nerves involvement (15). It is a rare condition (estimated $1-2$ cases per 100,000 person-years worldwide [16]. In approximately two-thirds of patients with GBS, an episode of acute infection precedes the neurological symptoms by $1-3$ weeks [17]. Infectious organisms recognized as potential triggers are diverse and include Campylobacter jejuni, cytomegalovirus, influenza viruses, herpes simplex virus (HSV), and human immunodeficiency virus (HIV).

Additionally, there is some evidence to suggest a temporal relationship between GBS and the receipt of various vaccines, including those for rabies, hepatitis (Hep) A and B, polio, and influenza [18]. Such cases are infrequent and the establishment of causality in most of these cases has proved controversial. However, a small increased risk of developing GBS specifically after influenza vaccination is relatively well established [19]. Recently there have been multiple case reports temporal relationship between COVID-19 vaccination and GBS development.

Our patient presented with bilateral upper extremity weakness and numbness four days following the vaccine. Initial evaluation revealed normal CSF studies and normal CBC, KFT, LFT. Nerve conduction studies were done, and they showed features of demyelinating disease. He was admitted to the hospital as a case of GBS. Following the diagnosis, he was started on Intravenous immunoglobulins for five days, which resulted in improvement of his symptoms on the following days. Which further confirmed the diagnosis. He continued to improve and was finally discharged with Follow-ups.

Patients with neurological findings should be enquired about recent vaccination history. It is of enormous importance, especially after the COVID 19 mRNA vaccine, which is newly introduced as it might have 
a link to the development of a widespread variety of neurological presentations. The risks of the vaccines are not fully understood; however, the benefits of the vaccines appear to outweigh the risks that might be encountered.

more patients gain access to the coronavirus disease

2019 (COVID-19) vaccines, neurologists are facing

questions about potential neurological complications, ben-

$\mathrm{e}$ ts, and timing of vaccination

The GBS/Chronic In?am-

matory Demyelinating Polyneuropathy (CIDP) Founda-

tion provides the following guideline: for the rare person

who develops GBS within 4 to 6 weeks of receiving an

immunization, it seems prudent to avoid that vaccination

in the future. For those whose GBS did not follow soon

after a vaccination, there are no reliable data to indicate

the risk of developing GBS after a vaccination.

10

The GBS/Chronic In?am-

matory Demyelinating Polyneuropathy (CIDP) Founda-

tion provides the following guideline: for the rare person

who develops GBS within 4 to 6 weeks of receiving an

immunization, it seems prudent to avoid that vaccination

in the future. For those whose GBS did not follow soon

after a vaccination, there are no reliable data to indicate

the risk of developing GBS after a vaccination.

10

The GBS/Chronic In?am-

matory Demyelinating Polyneuropathy (CIDP) Founda-

tion provides the following guideline: for the rare person

who develops GBS within 4 to 6 weeks of receiving an

immunization, it seems prudent to avoid that vaccination

in the future. For those whose GBS did not follow soon

after a vaccination, there are no reliable data to indicate the risk of developing GBS after a vaccination.

10

The GBS/Chronic In?am- 
matory Demyelinating Polyneuropathy (CIDP) Founda-

tion provides the following guideline: for the rare person

who develops GBS within 4 to 6 weeks of receiving an

immunization, it seems prudent to avoid that vaccination

in the future. For those whose GBS did not follow soon

after a vaccination, there are no reliable data to indicate

the risk of developing GBS after a vaccination.

10

The GBS/Chronic In?am-

matory Demyelinating Polyneuropathy (CIDP) Founda-

tion provides the following guideline: for the rare person

who develops GBS within 4 to 6 weeks of receiving an

immunization, it seems prudent to avoid that vaccination

in the future. For those whose GBS did not follow soon

after a vaccination, there are no reliable data to indicate

the risk of developing GBS after a vaccination.

10

conclusion :

COVID 19 pandemic has burdened many countries and created many changes in the physical, social and economic aspects. COVID 19 vaccines are an essential tool to help contain the pandemic. They have been proven to be very effective and safe, but at the same time, it is crucial to keep in mind the potential side effects of these vaccines. Patients who present with neurological complaints following the vaccine should be investigated for other causes first while keeping in mind that the vaccine can be a cause. As more patients are getting access to the coronavirus 2019 (COVID-19) vaccines, neurologists are facing questions about neurological complications, benefits and appropriate time of vaccination. GBS is one of the possible side effects that might be encountered after the COVID 19 vaccine. If it occurs, it appears to be responsive to the treatment, and the benefits of administering the vaccine outweigh the risks. The frequency of its occurrence is still needed to be studied.

Data availability statement:The data that support the findings of this study are available from the corresponding author upon reasonable request.

Conflict of interest: The authors have no conflict of interest to declare.

Funding information

Qatar national library - Qatar foundation

\section{Ethical Approval}

The case report was approved by Hamad medical corporation, MRC number MRC-04-21-387.

\section{Acknowledgments}

We thank the internal medicine department at Hamad Medical Corporation for giving us the opportunity and support to conduct this work. 
Authors Contribution:Ahmad S matarneh: History and physical examination, literature review, manuscript writing Alia Al battah: History and physical examination, literature review, manuscript writing Khalid farooqui Literature review, manuscript writing Mohammed ghamoodi: Clinical Care Mohammed Alhatou: clinical care, manuscript writing, literature review and mentor

References

1. Lu H, Stratton CW, Tang YW. Outbreak of pneumonia of unknown etiology in Wuhan, China: The mystery and the miracle. Journal of medical virology. 2020 Apr;92(4):401.

2. Esakandari H, Nabi-Afjadi M, Fakkari-Afjadi J, Farahmandian N, Miresmaeili SM, Bahreini E. A comprehensive review of COVID-19 characteristics. Biological procedures online. 2020 Dec;22:1-0.

3. Cascella M, Rajnik M, Aleem A, Dulebohn S, Di Napoli R. Features, evaluation, and treatment of coronavirus (COVID-19). StatPearls. 2021 Apr 20.

4. Yan ZP, Yang M, Lai CL. COVID-19 Vaccines: A Review of the Safety and Efficacy of Current Clinical Trials. Pharmaceuticals. 2021 May;14(5):406.

5. Kim JH, Marks F, Clemens JD. Looking beyond COVID-19 vaccine phase 3 trials. Nature medicine. 2021 Feb;27(2):205-11.

6. Lu L, Xiong W, Mu J, Zhang Q, Zhang H, Zou L, Li W, He L, Sander JW, Zhou D. Neurological side effects of COVID-19 vaccines are rare. Acta Neurologica Scandinavica. 2021 Jul;144(1):111.

7. Lotfi M, Hamblin MR, Rezaei N. COVID-19: Transmission, prevention, and potential therapeutic opportunities. Clinica chimica acta. 2020 Sep 1;508:254-66.

8. Yuan P, Ai P, Liu Y, Ai Z, Wang Y, Cao W, Xia X, Zheng JC. Safety, tolerability, and immunogenicity of COVID-19 vaccines: a systematic review and meta-analysis. medRxiv. 2020 Jan 1.

9. Anand P, Stahel VP. Review the safety of Covid-19 mRNA vaccines: a review. Patient safety in surgery. 2021 Dec;15(1):1-9.

10. Huynh W, Cordato DJ, Kehdi E, Masters LT, Dedousis C. Post-vaccination encephalomyelitis: literature review and illustrative case. Journal of Clinical Neuroscience. 2008 Dec 1;15(12):1315-22.

11. Kadali RA, Janagama R, Peruru S, Malayala SV. Side effects of BNT162b2 mRNA COVID-19 vaccine: A randomized, cross-sectional study with detailed self-reported symptoms from healthcare workers. International Journal of Infectious Diseases. 2021 May 1;106:376-81.

12. Goss AL, Samudralwar RD, Das RR, Nath A. ANA investigates: neurological complications of COVID19 vaccines. Annals of neurology. 2021 May;89(5):856-7.

13. Haber P, Sejvar J, Mikaeloff Y, DeStefano F. Vaccines and guillain-barre syndrome. Drug Safety. 2009 Apr;32(4):309-23.

14. Lunn MP, Cornblath DR, Jacobs BC, Querol L, van Doorn PA, Hughes RA, Willison HJ. COVID-19 vaccine and Guillain-Barre syndrome: let's not leap to associations. Brain. 2021 Feb;144(2):357-60.

15. Hughes RA, Cornblath DR. Guillain-Barre syndrome. Lancet. 2005 Nov 5;366(9497): 1653-66.

16 Sejvar JJ, Baughman AL, Wise M, Morgan OW. Population incidence of Guillain-Barre syndrome: a systematic review and meta-analysis. Neuroepidemiology. 2011;36(2):123-133. doi pubmed

17. Jacobs BC, Rothbarth PH, van der Meche FG, Herbrink P, Schmitz PI, de Klerk MA, van Doorn PA. The spectrum of antecedent infections in Guillain-Barre syndrome: a case-control study. Neurology. 1998;51(4):1110-1115. doi pubmed

18. Haber P, Sejvar J, Mikaeloff Y, DeStefano F. Vaccines and Guillain-Barre syndrome. Drug Saf. 2009;32(4):309-323. doi pubmed

19. Schonberger LB, Bregman DJ, Sullivan-Bolyai JZ, Keenlyside RA, Ziegler DW, Retailliau HF, Eddins DL, et al. Guillain-Barre syndrome following vaccination in the National Influenza Immunization Program, United States, 1976-1977. Am J Epidemiol. 1979;110(2):105-123. doi pubmed

more patients gain access to the coronavirus disease

2019 (COVID-19) vaccines, neurologists are facing 
questions about potential neurological complications, bene?ts, and timing of vaccination

more patients gain access to the coronavirus disease

2019 (COVID-19) vaccines, neurologists are facing

questions about potential neurological complications, ben-

e?ts, and timing of vaccination

more patients gain access to the coronavirus disease

2019 (COVID-19) vaccines, neurologists are facing

questions about potential neurological complications, ben-

e?ts, and timing of vaccination

more patients gain access to the coronavirus disease

2019 (COVID-19) vaccines, neurologists are facing

questions about potential neurological complications, ben-

e?ts, and timing of vaccination.
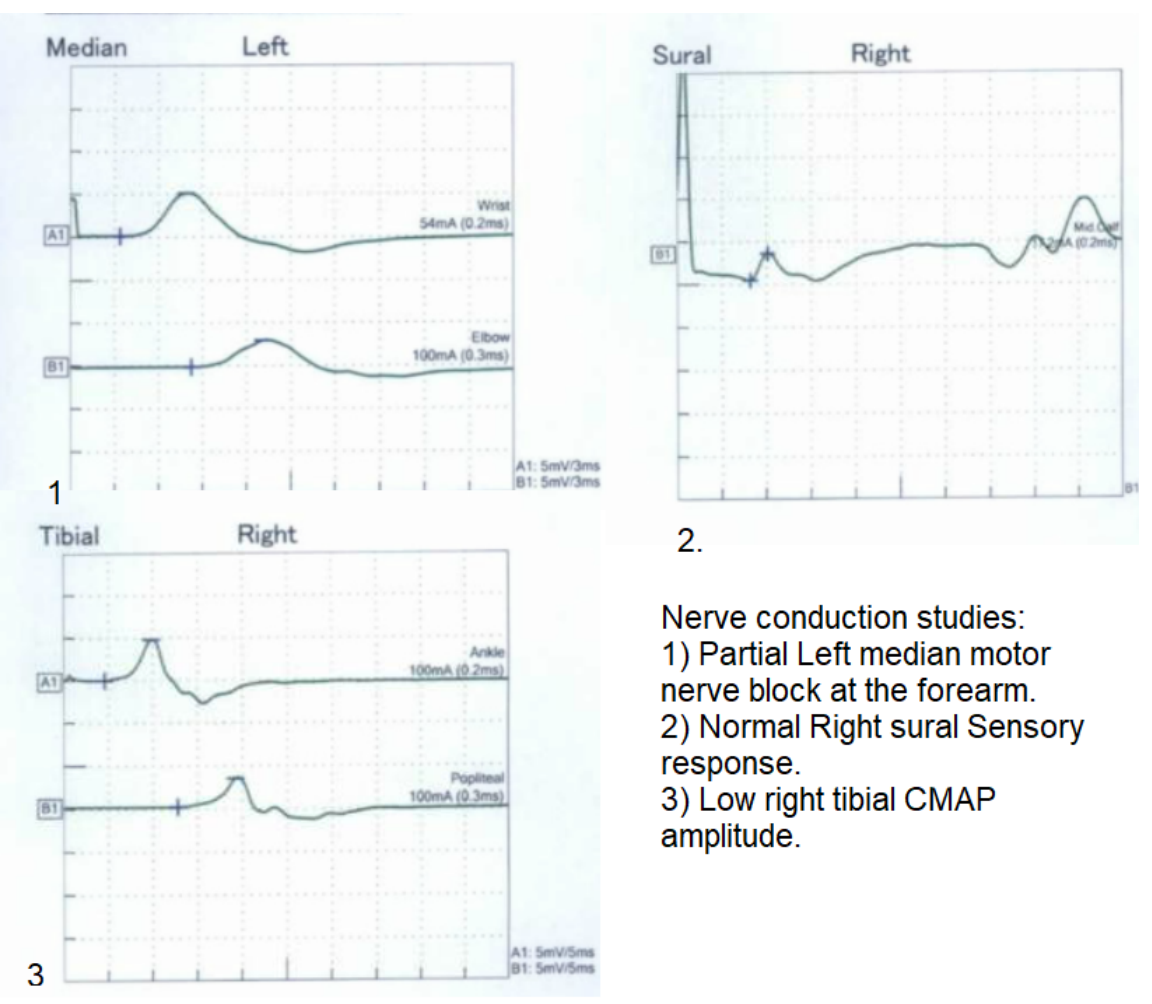

2.

Nerve conduction studies:

1) Partial Left median motor

nerve block at the forearm.

2) Normal Right sural Sensory

response.

3) Low right tibial CMAP

amplitude. 